13

\title{
Discussants comments on the ten main papers
}




\title{
Exotica/FMQM: A Persistent Message-Based Architecture for Distributed Workflow Man- agement - Discussion -
}

\author{
Stef Joosten and Sjaak Brinkkemper \\ Centre for Telematics and Information Technology \\ University of Twente \\ P.O. Box 217, NL-7500 AE Enschede the Netherlands \\ E-mail: \{joosten|brinkkemper\}@cs.utwente.nl
}

\section{SUMMARY}

This paper argues that most workflow management support systems are vulnerable due to the single database architecture upon which they are based. Furthermore, the single server architecture creates a performance bottleneck when large quantities of workflows are being managed.

To overcome this problem, the authors propose to store the knowledge in a distributed manner by means of persistent messages. The idea is that a message sent is never lost until it is received. In the proposed architecture, a node holds only the information needed to perform its part of the workflow. Every node holds a queue for each process that makes use of that particular node. These queues are implemented in stable store, thus providing the persistent functionality of messages. Each node maintains a process table for every process, based on its invocation by a process definition node. The process definition node may distribute a process over a number of nodes, each of which makes its own process tables. The process is kept together by a process thread. Instances are started, maintained and stopped by means of activity threads. An activity thread is started by a process thread. A nice feature is that instance tables need not be kept in stable store, because a crashed instance can always be recovered on the basis of the process tables (which are in stable store) and the current state of the queues.

\section{PERSPECTIVE}

This is the first detailed exposition of the distribution problems that are involved with workflow enactment. Most authors ignore the integrity problems associated with workflow, simply because they are handled by a single database implementation. The authors argue convincingly that this conventional workflow architecture will not stand up to the needs of large scale workflow systems. 
The idea to use persistent stores at the individual nodes is new. The paper is well written and clearly understandable by researchers with a little knowledge of workflow in general and FlowMark in particular.

\section{CRITIQUE}

There is no evidence as yet that the proposed architecture works. So far, only the ideas have been described.

This work has many similarities with the design of the Job Transfer and Manipulation protocol (JTM; see l'Anson \& Pell, Understanding OSI Applications, Prentice Hall, Englewood Cliffs, 1993. chapter 13). However, the relation with JTM is not mentioned.

The use of the reference model of the Workflow Management Coalition is rather obvious. That model is superficial enough to accommodate any serious workflow architecture.

The work leads to so many different consequences that all need research, that the section on future work is unreasonably shallow.

\section{QUESTIONS}

Is it possible to prove the stability of the workflow system, based on the assumption that node/process queues and process tables are persistent? Has this proof been done?

The removal of messages from queues implies that copies of messages are made in case different activities share a message. Does this lead to redundancy? Performance loss?

How is the aggregation of managerial information and statistical data of running workflow applications at runtime nodes being supported in the sketched architecture? The distribution architecture seems to require extensive querying of all runtime nodes. 


\title{
The Systems Integration Architecture: An Agile Information Infrastructure - Discussion -
}

\author{
John Krogstie \\ IDT, NTH \\ N-7034 Trondheim \\ Email: johnkrog@idt.unit.no
}

\section{DISCUSSANT'S SYNOPSIS}

The Systems Integration Architecture (SIA) project claim to take a fresh approach to integration with special emphasis on supporting Agile, Virtual enterprises and special need for reconfigurable, de-centralized information systems. Agile Manufacturing is an approach in which manufacturers must be able to rapidly respond to unanticipated market demands, producing new products with resources outside of their immediate company in so-called Virtual Corporations. In these, complementary core competencies from diverse companies including suppliers and customers, are combined temporarily into a new, decentralized company existing only to fulfill that market demand and dissolving when the demand is satisfied. Even if a company is not ready to become Agile, to survive in today's competitive world, it must be continually seeking to improve itself. Such continuous improvement requires change, often rapid change.

To address this situation, the authors propose a workflow modeling and enactment system based on the IPO (Input-process-output) approach. Processes can be manual, computer aided, or automated. In the two first cases one also indicate the human actor of the process. The input and output from processes have a type, a data format, a file structure, and an access type. Control flow is also possible to indicate. Around this partly traditional language, the system integration architecture is built up, providing services in data management, control, and communications. SIA uses the concept of processes to allow the user to define a workflow by checking for the appropriate format for the tool integration through a special user-interface. SIA also keeps track of the information interfaces through a data management system via a communication kernel based on CORBA. SIA is currently being implemented in $\mathrm{C}++$ on a several UNIX-platforms.

\section{POINTS FOR DISCUSSION}

We will below raise some points for discussion based on our own background.

- Generally, we feel that more references to literature within conceptual modeling and comparisons with this kind of work could be provided. Reading the paper, I see a clear 
connection to work within many different perspectives in the conceptual modeling field, e.g. the structural (data) perspective, the functional (process) perspective e.g. Ward/PPP, the behavioral perspective, the organizational(actor/agent/role) perspective e.g ALBERT and the communicational (speech acts) perspective e.g Action Workflow.

- Within workflow modeling, it is usual to differentiate between two main approaches, the IPO approach, which the article present, and the language action approach. The IPO approach is usually proposed for use in stable environments whereas the language action approach is proposed for use within changing environments. The article proposes an IPO approach for rapidly changing environments. Have you looked upon the language action approach?

- The matching mechanisms that are proposed support matching on a syntactic level by matching types and modalities, but not on a semantic level. When having a fully automated process being "a rule based design system which transforms a set of specifications into a full design according to the rules embedded in it" there is a need for a semantic matching being also based on the content and meaning of the data. As far as I can see, there is no support for this in the framework, an area which will be especially problematic when putting together virtual organizations based on parts of organizations which inevitably will have a different culture, use partly different terminology etc. Here one might find inspiration within work on view integration and reuse of conceptual models (see e.g. Francalanci and Shanks for an overview of these fields).

- The article refer $t$ oan implementation effort of the framework How far has the implementation come?

- Do you have any experiences with the practical use of the framework? 


\title{
Message flow control - Discussion -
}

\author{
Atika Laribi and Michel Leonard \\ Centre Universitaire d'Informatique,Universitat de Geneve \\ 24, rue du General-Dufour, CH-1211 GENEVE 4 \\ Switzerland \\ Email: \{laribi|leonard\}@cui.unige.ch
}

This paper presents a framework for the development of a message control system, an issue which is at the heart of Decentralized Information Systems implementation. Even though the major IS concept of business transaction is presented as being the cornerstone of the system, it is not thoroughly explored. The authors stay at a rather technical level and fail to fully explain the implications of their approach on Decentralized Information Systems. However, by staying at this level, the authors are able to bring to our attention a fundamental issue namely the control of message exchanges. The paper therefore, changes its focus from business transactions to transaction protocol handler. This handler coordinates the exchange of messages between actors. One has to be careful with terminology here, as actor is defined in a purely technical context and does not necessarily refer to the usual Information System actor. For the benefit of the DBMS community, a quick analogy is made with database transactions. Even though business transactions have all the ACID properties, one way they differ significantly from their database counterpart is the fact that they may be carried out by humans. Although database transactions management has been extensively studied, a model specific to business transactions is required to cater for the differences. Business transactions can have a superior and subordinates. A given transaction T1 can be both the subordinate of a transaction T2 and the superior of a transaction T3. ExSpect (Executable Specification Tool) is used to implement the transaction state transition diagram representing the system architecture. The paper lays the ground for the development of a useful analysis tool for Information Systems managers. The prototype presented can be directly used to simulate the interactions between a given transaction and the transactions it needs to cooperate with to complete a given activity. The coordination revolves around the control of the message flow between the different cooperating transactions. This is indeed an important feature of the prototype since the only way business transactions communicate is through messages. 


\title{
A light weight name service and its use within a collaborative editor - Discussion -
}

\author{
Patrizia Asirelli \\ Instituto di Elaborazione dell' Informazione del C.N.R., \\ Via S. Maria, 46-56126 Pisa, Italy.- \\ Tel.+39-50-593477 \\ Email: asirelli@iei.pi.cnr.it
}

\begin{abstract}
In this short discussion on [LUPA95] I shall point out a tendency in the evolution of an area of research that suggests interesting, in my opinion, extensions to the ideas presented in that paper. Furthermore, I am going to suggest the exploitation of the Light Weight Name Service, eventually extended, within the framework the World Wide Web.
\end{abstract}

\section{INTRODUCTION}

First I shall introduce few notions of Software Configuration Management (SCM for short) that immediately suggest which extensions could be made to the LWNS proposal and why those extensions would be useful. Then I shall briefly point out other similarities with the World Wide Web that I am certain the authors have considered and point out which advantages the LWNS, eventually extended, would bring into that area.

\section{SOFTWARE CONFIGURATION MANAGEMENT AND ITS RELATION TO THE COLLABORATIVE EDITING PROBLEM}

As the author says in [LUPA95] their LWNS proposal has the basis for further extensions and, in my opinion, the trend in the Software Configuration Management (SCM for short) area [WSVCC88], [WSCM91], [ESEC87],[ICS95] [FEL79] [TRENDS94] should be considered. The reason for this is the similarities it shows with the DUPLEX environment for which LWNS was designed while, on the other hand, I believe the SCM area could take advantage and suggestions to deal with "distributedness". Configuration Management refers to the activity that controls software evolution. The basic idea is that a software system is built up by putting together pieces (modules) of software that are developed by different programmers, may be at different time. At present, given the "distributedness" of many of the environments on which we work, the developers of a module may be resident on a different site and he/she may be also using a different tool and computer. Still, the development of the system is an activity that is collaborative, thus there must be a manager. Thus, the SCM activity basically deals with two main concepts: the system model that describes the architecture of the software system 
and the software components (objects) or system family. Software components themselves are objects that may be obtained by collecting together objects and so on. Everything becomes even more complex when considering "reuse" and multiple "versions" of objects. Versions may be "variants" (different implementation of the same "specified function") or "revisions" (evolution of an object) that may be plug into the system to lead to a different version of the system itself. The configured system is a collection of all the software components. Thus to build a configuration it means to assemble objects with certain characteristics. Characteristics are properties of objects for which one version is chosen rather than the other, e.g. the modification date [FEL79], etc. Summarizing, SCM environments need:

- a file system where objects can be found;

- strategies to choose the objects in the file system.

The actual trend in SCM is that configurations can become very sophisticated and the process of building a system version can become more powerful if more knowledge on the objects is put into the file system, thus, for example it could be possible to build configurations not only with objects that have a certain creation/modification date[FEL79], but also on the basis of the "author's name/group" or whatever else one might think it necessary and possible to say on objects. This trend is thus asking for evolution of the simple file system to be a database[TICHY85, TICHY88, ESTUB86, ESTUB94], and not just a relational one but, for example, Object-Oriented[ATKI89, DEUX91, ESTUB94], Deductive [ZAN90, ASI87a, ASI87b, ASI88, AQUI91, AQUI92] or a knowledge base in general. Thus the tendency is to give more structure to the information on the objects and on strategies and inference rules; on deductive capabilities in general so to be able to make some form of reasoning The similarities with the collaborative editing environment (DUPLEX), suggest that a name server for it should grow towards the above kind of knowledge base. Thus the suggestion is to investigate into an evolution of the LWNS towards a more flexible structure for the object references. that would permit to say more than just: 1) the address of the service; 2) the access protocol;3) the object's local name. This probably would also mean that all the LWNS should have to be reconsidered e.g. the prefix table should become a database etc.. The efficiency of such an approach should be evaluated to find a fair compromise between fast responses and complexity of the query to find objects in the collaboration (or in the Web, see next section).

\section{LWNS WITHIN WWW?}

The World Wide Web is overwhelming every one of us and this Conference certainly sees an interested and competent audience. Although not all of us may be directly involved in working for the Web, in the sense of producing documents to be put on it or producing tools for it, I am fairly certain that every one of us is, by now, aware of how things work and how to navigate within WWW. It is from this very little and naive experience that while reading the paper [LUPA95] a question naturally emerges: such a naming service could be adopted as the name service within WWW. Again, I would like to point out the similarities between the environments for which the LWNS was designed and the WWW. There is collaboration among users connected trough Internet, as in WWW. Long distance between users. Users that work on the same documents or use documents produced by other users. There is also the same kind 
of approach in constituting a collaboration. Users may be added, deleted and their reference modified. There must be a manager of the collaboration (the Web node: ftp, http, etc.). Thus there seems to be no reason why one should not investigate into the use of such a name service within WWW. The advantage for the WWW community would be, in my opinion, that there would be more flexibility in the creation/modification/deletion of objects and more powerful retrieval operations could be performed on the net. On the other hand, extending the LWNS in the direction mentioned in the previous section, and then integrating it into the Web, would bring into it some form of reasoning with all the advantages previously mentioned.

\section{CONCLUSION}

I would like to conclude this discussion mentioning that, from the documentation I have found on the Web [WWW,URL], I see a tendency to extend the actual UR system towards the direction discussed here. That is, as far as I can see, the evolution proposed for LWNS considering the trend in the Software Configuration Management, and the integration (or use of it) within the WWW, would exactly be coherent with the tendency shown in the extension of the actual naming system of the WWW [URL]. Now a question arises: the UR system can be extended and made soon freely available. This is a fear expectation considering how fast this area has grown in very little time, then: wouldn't the LWNS become quickly obsolete? I am sure that the authors of [LUPA95] had already made the considerations I have made in this discussion and will present their own opinion on the matter.

\section{ACKNOWLEDGMENTS}

I would like to thank Keith Jeffery, Arne Sølvberg and the organizers of the ISDO'95, for giving me the opportunity to learn something new and to write this paper. I also thank my colleague Dr. Franco Mazzanti for all the useful and stimulating discussion on these ideas.

\section{REFERENCES}

[ASI87a] P. Asirelli, P. Inverardi - Enhancing Configuration Facilities in Software Development: A Logic Approach, Proc. of ESEC - 1st European Software Engineering Conference , Strasburg, 8-11 Sept., 1987, Lecture Notes in Computer Science, No. 289, Springer-Verlag 1987.

[ASI87b] P. Asirelli, P. Inverardi - A Logic Database to Support Configuration Management in Ada, Proc. Ada Europe International Conference, Ada Components: libraries and tools, Stockholm, 26-28 May, S. Tafvelin (ed.), The Ada Companion Series,Cambridge Univ. Press, 1987.

[ASI88] P. Asirelli, P. Inverardi - EDBLOG: a kernel for configuration environments, Proc. Int. Workshop on Software Version and Configuration Control, J.F.H. Winkler (ed), Grassau, B.G. Teubner Stuttgart, 1988, pp. 422-425.

[ATKI89] Atkinson M.P., Bancilhon F., DeWitt D., Dittrich K., Maier D. and Zdonik S., The Object-Oriented Database System manifesto, Proc. DOOD 1989, Kyoto, Japan, pp. 40-57. 
[AQUI91], D. Aquilino, P.Asirelli, P. Inverardi, P. Malara - Supporting Reuse and Configuration: A port based SCM model; Proc. of the 3rd Int. Workshop on Software Configuration Management, J. Feiler Ed. , 12-14 June1991,Trondheim - Norway, ACM Press.

[AQUI92] D. Aquilino, P.Asirelli, P. Inverardi - Maintaining Knowledge in System DevelopmentAbstract to be presented at the Fourteenth International Conference On Software Engineering (ICSE14) -Melbourne, Australia, May 11-15, 1992.

[DEUX91] Deux O. et al., The O2 System, Communication of the ACM, Vol. 34, N. 10, pp34-48.

[ESEC87] Proc. of ESEC - 1st European Software Engineering Conference, Strasburg, 8-11 Sept., 1987, in LNCS 289, Springer-Verlag 1987.

[ESTUB86] Estublier, J. Adele: a data base of programs. Presentation manual, Laboratoire de Genie Informatique, France, June 1986.

[ESTUB94] Estublier, J., Casallas, R., The Adele Configuration Manager, Adele: a data base of programs. Presentation manual, Laboratoire de Genie Informatique, France, June 1986.

[FEL79] S.I. Feldman, "MAKE - A Program for Maintaining Computer Programs", Software Practice and Experience 9, pp. 255-265 (April 1979).

[ICS95] Workshop on Configuration management of the 17th ICSE, International Conference on Software Engineering.

[LUPA95]J.-C. Lugeon and F. Pacull, A Light Weight Name Service and its use within a collaborative editor. In Proc. of the IFIP WG8.1 Working Conference on Information System Development for Decentralised Organisations-ISDO'95, Chapman \& Hall, 1995.

[TICHY85] Tichy, W., RCS- A System for Version Control, Software Practice and Experience 15,7 (July 1985), 637-654.

[TICHY88] Tichy, W., Tools for Configuration Management, Proc. Int. Workshop on Software Version and Configuration Control, J.F.H. Winkler (ed), Grassau, B.G. Teubner Stuttgart, 1988. [TRENDS94] Trends in Software: Configuration Management, W. Tichy Ed., John Wiley\& Sons, 1994.

[URL] http://www.ncsa.uiuc.edu/demoweb/url-primer.html or http://www.w3.org/hypertext/WWW/Addressing/Addressing.html

[WSCM91] Proc. of the 3rd Int. Workshop on Software Configuration Management, J. Feiler Ed. , 12-14 June1991,Trondheim - Norway, ACM Press.

[WSVCC88] Proc. Int. Workshop on Software Version and Configuration Control, J.F.H. Winkler Ed., Grassau, B.G. Teubner Stuttgart, 1988.

[WWW] http://www.w3.org/hypertext/WWW/TheProject.html

[ZAN90] Zaniolo C., Deductive Databases: Theroy Meets Practice,(Invited Paper) Proc. EDBT'90, Lecture Notes in Computer Science, No. 416, Springer-Verlag, pp. 1-15. 


\title{
Organizational management system in a heterogeneous environment: A WWW case study \\ - Discussion -
}

\author{
Martin Bever \\ IBM Deutschland Informationssysteme $\mathrm{GmbH}$ \\ European Networking Center \\ Vangerowstrasse 18 \\ D-69115 Heidelberg, Germany \\ Email:mbever@vnet.ibm.com
}

\section{INTRODUCTION}

Since the introduction of the World Wide Web, this technology has experienced an unforeseen growth. A significant portion of the overall traffic found within the Internet now is due to WWW, and it is still heavily growing. This clearly indicates WWW is solving a problem users have all around the world, namely having easy access to all the informations present within different places.

On the other hand, one also can see the proliferation of new problems arising from this approach, namely how to find the right information in reasonable time, and how to ensure what one gets is still up to date (no way to deduce this from WWW pages).

\section{THE REPORT}

The paper written by Silva, Borbinha and Delgado reports about using the WWW technology to give users easy access to a multitude of data management systems (e.g. file systems or data base systems), storing informations in a variety of formats often different from HTML. A gateway is used to map both the format of the content to the HTML format exchanged within WWW, and the functionality provided by the data base to HTTP In this way, it becomes possible to read documents in multiple formats using a WWW client. Since these clients are available on many platforms, this approach conveniently provides access to all informations within a heterogeneous environment.

However, since each data base uses a specialized format and access technique, a corresponding specialized gateway process is required. This clearly makes sense if lots of documents that have to be kept in a special format shall be made available to WWW users. If, however, an information service about an organization has to be developed from scratch, why not writing these 
pages directly in HTML? Extending these considerations, one might ask the question whether HTML is the solution to the problem how to describe the contents of a sheet. We have seen other approaches (like ODA) in the past, and new ones still are evolving (like e.g. the Portable Document Format, PDF). Open issues are e.g. the extension of HTTP to allow real-time access to multimedia data. Finally, finding the right information in time would become much easier if a rich search function were available in HTTP, like e.g. in the Document Filing and Retrieval (DFR) protocol.

\section{IMPACT OF VVWW TO TELECOOPERATION}

Extending the scope of the paper, large organizations have to find solutions how their different sites (mostly located all around the world) can efficiently interact among themselves and with their customers. It is the goal of telecooperation to increase the efficiency of any collaboration between parties located at different geographical sites even across country borders.

Making information available in the appropriate form to a multitude of users clearly is one important aspect in this area. The paper restricts itself on accessing documents in an off-line mode, i.e. retrieving it page by page from a repository and reading it within a WWW client. However, a perhaps even more important scenario is the synchronous interaction among groups of people within a desktop conference.

Here, the cooperative viewing of documents is a topic of main interest. It should be possible to view the document in a synchronous manner, to add annotations to it and so on. A final question that is worth further discussion is about the impact the WWW technology can have on telecooperation, and the perspective of the technology in this rapidly growing, highly interesting area. 


\title{
A federated information system for quality management processes - Discussion -
}

\author{
Jan l. G. Dietz \\ Delft University of Technology, \\ Faculty of Technical Mathematics and Informatics \\ Information Systems Group \\ P.O.Box 356, 2600 AJ Delft, The Netherlands \\ E-mail:dietz@is.twi.tudelft.nl
}

\section{DISCUSSANT'S SYNOPSIS}

A key question for the field of information systems in the context of business process (re)engineering, which leads to networks of working groups integrated by material and information flows, is whether the latter imposes new practices for the (re)design and (re)engineering of the information systems supporting the business processes, and if so, which ones. The authors address this question by contending that a new information systems architecture is needed in order to fulfill the requirements imposed by a continuously changing and flexible business environment. This new architecture then should be repository-based and federated.

Although the basic technology (computer networks, repositories and access mechanisms) to implement such an architecture is available, there are a number of problems still to be solved. Three of these problems are indicated and discussed in the paper:

- The conceptual distance between different working groups.

- The task-oriented access to and availability of information.

- The management of change of an installed information system.

According to the authors these problems can be solved adequately if information systems are based on the Trader architecture, which is the outcome of the WibQuS project on the integration of quality management methods in which all of the authors have participated.

The problem of the conceptual distance is addressed by the hierarchical layering of the conceptual schema in the Trader architecture, based on a common repository meta model. The architecture allows for distinguishing between global schemas and private schemas.

The problem of information access and availability is addressed by the design of an abstract request language. A request is formulated by a predicate and an argument list. This is transformed automatically to SQL-statements appropriate for the DBMS that controls the information base containing the information searched for. In addition, a task and view oriented information system browser is proposed in order to meet the need to explore the (federated) repositories. This kind of queries are formulated in a graphical way. 
In order to address the problem of management of change, the authors propose to use the Trader functionality itself to conceptualize changes and eventually adjust databases. This proposal is not elaborated however.

\section{DISCUSSANT'S CRITICISM}

Although the paper is clearly structured, I found it rather hard to read for two reasons. One of them is that conceptual issues (specification) and technical issues (implementation) are mixed up, especially in section 3 . The other one is that the figures are badly explained, and next to that contain errors. A serious error is contained in figure 1. If I understood the accompanying text well, then there are not three but two levels of abstraction: both QFD and DACAPO are instances of the type Method. At best DACAPO could said to be included by QFD (a relation type that is defined at the meta level), but DACAPO definitely cannot be an instance of QFD. A minor, but nasty error is contained in figure 3. To my knowledge, the last line of the query template should read: " $\{1\}\{1\}$ MR. $\{1\} M=\{1\}$ M.ID".

Although the paper draws heavily on the notion of task and its relationship to information need and information production, this attention appears to be nothing more than lip service, because the notion is not defined. It is the discussant's conviction that as long as notions like business process, task, communication and information remain not exactly interrelated, more or less axiomatic, notions, one cannot expect much progress in the field of information systems design \& engineering, whether or not in the context of business reengineering. In this respect I like to bring to the authors' attention recent work that is rather successful in providing a profound and integrated understanding of these notions (see references below).

\section{BIBLIOGRAPHY}

[1] Medina-Mora, R., Winograd, T., Flores, R., Flores, F., The Action Workflow approach to workflow management technology, in: Turner, J., Kraut, R. (eds.) Proceedings of the 4th Conference on Computer-Supported Cooperative Work, ACM, 31 october- 4 november, Toronto, 1992.

[2] Taylor, J.R., Rethinking the theory of Organizational Communication, Ablex Publishing, Norwood, N.J., 1993.

[3] Dietz, J.L.G., Busines Modelling for Business Redesign, Proceedings of the 27th Hawaii International Conference on System Sciences, IEEE Press, 1994.

[4] Dietz, J.L.G., Modelling business processes for the purpose of redesign, in: IFIP Transactions A-54 Business process Re-engineering, Information Systems Opportunities and Challenges, North-Holland, 1994. 


\title{
Modelling business relationships in a non- centralized systems environment - Discussion -
}

\author{
Panos Constatopoulos \\ Institute of Computer Science FORTH \\ Science and Technology Park PoB. Box 138 \\ GR-71110 Heraklion, Crete,Greece \\ Email:panos@ics.forth.gr
}

In this paper Macdonald and Veryard present a methodology for modelling business relationships to support information system development in a decentralized, eventually federated, open setting.

Federation and openness are viewed from three prespectives : technical, organizational/ managerial and market/entrepreneurial. The authors discuss the issues involved in designing and implementing open, federated systems. In particular they address organizational issues, such as incremental development; systems and information crossing organizational boundaries; the effects of interaction distance on resource sharing, communication between agents and coordination between activities; determining requirements in view of multiple requirements ownership and organizational learning; and connections among organizational, technical and commercial aspects.

On the premise that openness essentially concerns federated computing accross organizational boundaries, the authors adopt organizational structure and boundaries as the perspective from which to model business relationships. In view of the organizational issues examined, this can offer useful support to the development of an open information system.

Although intuitively quite clear and well illustrated by the case study, the proposed methodology lacks formality and completeness. Underlying the four zones of organizational intervention (fig. 5) and the eight methodology tasks (fig. 6) there are a number of basic concepts (e.g. agent, interaction, activity, task, perspective) as well as a set of related axioms and rules concerning the construction of models. Some degree of formalization, providing precise semantics, would be necessary for the applicability of the methodology and eventual incorporation in computersupported development environments. Furthermore, it will be very interesting to see a complete definition of the methodology including the implementation and learning zones. Implementation refers to organizational implementation. The authors view this as a process of change and introduce a vague notion of "problem solving engine" to address implementation and delivery problems.

Instrumental in the proposed methodology is the notion of 'interaction distance'. An intuitive notion, clearly illustrated in the case study, yet hard to make operational. They key to this effect would be the precise definition of interaction distance as a distance measure in an appropriate space, along with a procedure for computing it. Until such a definition becomes available, only illustrative use of the notion will be possible. 
The applicability of the methodology to the development of federated systems relies on the explicit consideration of boundaries. It will further be interesting to investigate the nature of federated development of systems and the applicability of the business relationship modelling methodology in that case. 


\title{
Realization of a decentralized IS-strategy - Discussion -
}

\author{
Rudolf Andersen \\ IDT, NTH \\ N-7034 Trondheim \\ Norway \\ Email: rudolf@idt.unit.no
}

The paper presents a theory of an IS-strategy which focus on facilitating operation and cooperation of decentralised, function oriented and autonomous information systems designed to support independent business functions. This is a topic of great importance to contemporary businesses. Current trends are towards reengineering the business into independent profit centres and organising the work into lean work processes. Together with an increasing rate of change in the environment, the need for flexible information systems support to provide cost-effective solutions has not been reduced. The survivers in this climate are the businesses which manage to maintain adequate information systems support through the storms of organisational reshuffling. An important part of this is to maintain an information systems architecture which is modification friendly, and which supports rather than constrains the core business of the organisation.

The paper presents a theory which focus on autonomy of information systems. A strong link between responsibility of business functions and responsibility of information systems is emphasised. And the information resources of value to the whole business, are provided to the business functions through a functionally oriented information system. The theory is presented and discussed in an informal way. The IS-stategy theory presented includes several concepts/phenomena which have been heavily investigated in the IS literature, e.g., user involvement, management involvement, and user attitudes towards IS. Thus, one would expect to find more extensive references to the literature.

The analysis of the theory is supported by a case study of three different organisations employing alternative strategies for decentralising their information systems. This empirical approach potentially provides for very interesting results. The fact that this is a real life case study, means that the findings and conclusions have an interest to a number of organisations. However, we are told very little about the case study method. The organisations taking part in the case and the actual findings of the case are also described in very little detail. This means that the analysis of the theory presented and the conclusions drawn from the findings in the case study have limited credibility and value to the reader. In this respect it would have been better not to abstract so much, but rather give actual statements from the interviews. 


\title{
A software process model for business reengi- neering \\ - Discussion -
}

\author{
T. William Olle \\ T. William Olle Associates LTD \\ 2 Ashley Park Road \\ KT12 1JU Walton-on-Thames, Surrey, United Kingdom \\ Email:100010.3176@compuserve.com
}

\section{OVERVIEW}

The main aim of this paper appears to be to attempts to "bring together" earlier pieces of work from different sources namely

- SPM, the software process model by the authors

- BRP, the business re-engineering plan

- CMM2, level 2 of Capability Maturity Model by SEI

The aim of the bringing together appears to be more for comparative analysis rather than synthesis.

\section{BUSINESS RE-ENGINEERING}

The frequent use in the paper of the term "business re-engineering" is somewhat misleading as the main thrust of the paper is towards a "software process model", in other words a model of the software process which should be used to develop the software to support the business processes. Business re-engineering is surely aimed at improving in the way the business processes are performed, this improvement being based on the availability of a computerized information system (which the paper persistently refers to as "software").

\section{PARTS OF ENTERPRISE MODELLING}

One has to look at Figure 3 to realize that the SPM and the SISU Enterprise Model are extremely close. One of the more useful features of the paper is the description of the SISU enterprise model although, as described, it would be better referred to as an "enterprise modelling facility" (a term used once in the text. The term "enterprise model" should be restricted to its more 
frequent and more intuitive use for referring to a model of an enterprise and not for referring to " a way of modelling enterprises".

Nevertheless, the breakdown of enterprise modelling (in Figure 1) identifies the following parts: 1) configuration, 2) objectives, 3) activities and usage, 3) concepts, 4) actors, 5) functional requirements, 6) non-functional requirements, 7) information system, is quite useful, although it is not clear why everything has to be called a "sub-model" when it is clear it is a modelling facility. It is also not clear why the information system has to be positioned as part of enterprise modelling. Surely enterprise modelling is about developing an analytic model of the enterprise. On the basis of this analytic model, the computerized information system can be designed and the design can be regarded as prescriptive model.

One must also question whether non-functional requirements (as described in the paper) are actually part of enterprise modelling. They should preferably be positioned as part of the prescriptive statement about the computerized information system.

Another quibble concerns the interpretation of the "concepts sub-model". As described in terms of object types, relationships and object properties, it seems to be restricted to nothing more than good old fashioned data structuring (sometimes referred to as information structuring).

\section{CONFIGURATION MANAGEMENT AND TRACEABILITY}

It is good to see configuration management and version control mentioned albeit only in the context of enterprise modelling. The orthogonality of configuration management to other parts of enterprise modelling is unquestioned. However, the all important concept of traceability should have been mentioned in this context as it is one of the more important reasons for needing configuration management.

In simple terms, it is necessary to be able to trace the capabilities provided in a computerized information system back to the requirements established in the model of the enterprise and to be able to trace the requirements established in the model of the enterprise forward to the capabilities provided in the computerized system.

\section{CONCLUSIONS}

It would be surprising if a conclusion of this paper were that the three pieces of work compared in the paper were other than incomplete and disparate. Each of the three has a different perspective on the overall information systems life cycle. One can also argue that problems related to project management, important though they are, can usefully be separated from the essentially technique work being performed in a technical project.

Nevertheless there must exist a set of fundamental concepts and it might be helpful for teaching and for practice if researchers could achieve some consensus one what these concepts are. 


\title{
Coupling process models and business rules - Discussion -
}

\author{
Colette Rolland \\ U.F.R. O6 - Gestion Universite de Paris 1, Pantheon Sorbonne \\ 17, Rue de la Sorbonne \\ F-75231 Paris Cedex 5,France \\ Email: rolland@masi.ibp.fr
}

The paper presents a nice, simple technique for relating a process model to a rule model. The process model is an extension of the Gane\& Sarson's data flow diagramming technique which distinguishes between control and data flows and introduces the notion of AND, OR and XOR ports. The rule modeling language (ERL) allows to express rules in the form when (trigger) if (condition) then (consequence) to constraint static or dynamic aspects of the conceptual schema. Both models have been developed within the ESPRIT project TEMPORA. The paper clearly shows the correspondences between the process model and the rule model and show how change in one model can affect the other. Rules have been demonstrated to be an important aspect of conceptual modeling. However little effort has been paid to the methodological dimension of rule elucidation and formalization. By introducing the idea to derive business rules from data flow diagrams, the authors of the paper contribute significantly to this field. Moreover, the careful study of the impact of change in one model to another is a useful contribution to system evolution and reengineering. However it is not stated whether the models have been used in practice for business reengineering even though it is claimed that this is the purpose. The acceptance of the models in a professional arena and their actual usefulness have not been validated. The appropriateness of the approach in a distributed context remains to be demonstrated. But the paper presents a solid piece of work and an interesting and good mapping between a process model and a rule model. 